\title{
Direct Observation of Two-Dimensional Electron Solvation at Alcohol/Ag(111) Interfaces
}

\author{
S. H. Liu, A. D. Miller, K. J. Gaffney, ${ }^{\dagger}$ P. Szymanski, S. Garrett-Roe, I. Bezel, ${ }^{\dagger}$ and \\ C. B. Harris* \\ Department of Chemistry, University of California, Berkeley, California 94720, and Chemical Sciences \\ Division, E.O. Lawrence Berkeley National Laboratory, Berkeley, California 94720
}

Received: March 16, 2002; In Final Form: October 7, 2002

\begin{abstract}
Time-resolved two-photon photoemission was used to investigate the two-dimensional electron solvation by methanol, 1-propanol, 1-butanol, and 1-pentanol overlayers on a $\mathrm{Ag}(111)$ surface. For each system at coverages higher than one monolayer, several image potential state series with time-dependent energies were observed by using two-photon photoemission indicating that multiple time-dependent local work functions can originate from multiple coverages. The time-dependent energy relaxation seen is attributed to the rotation of the adsorbate molecular dipoles to solvate the electron. This rotation lowers the electron-layer interaction energy, causing a dynamic reduction of the local work function which is a signature of the solvation of the electron by the adsorbate layer. A classical electron-disk dipole model supports the conclusion that the dynamic variation in the image potential state energies results from a local work function effect and indicates that the evolution of the energy results from the time-dependent projection of the molecular dipole onto the surface normal on the femtosecond time scale.
\end{abstract}

\section{Introduction}

The dynamics of molecular motion on metal surfaces is of central importance for an understanding of microscopic physical and chemical processes that occur at a surface, e.g., island growth, molecular diffusion, and catalysis. The dynamics of these processes can be complicated by the presence of an electron at the surface. A strong electron-adsorbate interaction can cause an electron induced adsorbate reorganization ${ }^{1-3}$ similar to electron solvation in liquids. ${ }^{4-6}$ In liquids, electron solvation dynamics are known to critically influence the rates of a wide variety of processes, including vibrational relaxation, ${ }^{7-9}$ ion transport, ${ }^{10}$ molecular isomerization, ${ }^{8,11}$ protein folding, ${ }^{12}$ and electron-transfer reactions. ${ }^{13-15}$ Studies of solvation at an intrinsically asymmetric environment such as an interface is particularly interesting because the reduced dimensionality and hindered solvent motion can result in dynamics distinct from those in the isotropic material. ${ }^{16-19}$ The present report describes a novel application of time-resolved two-photon photoemission (TPPE) to the study of two-dimensional solvation dynamics at polar solvent/metal interfaces on the femtosecond time scale.

Time-resolved TPPE is a pump-probe technique where an initial pump pulse excites electrons from occupied metal bands to unoccupied interfacial states, and after some delay time $\Delta t$, a probe pulse photoemits the interfacial electrons into the vacuum (Figure 1). The final kinetic energy and TPPE signal intensity of the photoemitted electrons provide a direct measure of the excited-state energy and population dynamics. ${ }^{20-26}$ The present report demonstrates that the photoelectron kinetic energy dynamics reflect the reorganization of molecules adsorbed on a metal surface. We present results for methanol, 1-propanol, 1-butanol, and 1-pentanol adsorbed on a $\mathrm{Ag}(111)$ surface, though similar results have been seen for nitrile adsorbates. ${ }^{2}$ A model

\footnotetext{
* Corresponding author. E-mail: harris@socrates.berkeley.edu.

$\dagger$ Present address: Department of Chemistry, Stanford University, Stanford CA, 94305.

$\doteqdot$ Miller Institute for Basic Research in Science, 2536 Channing Way No. 5190, Berkeley, CA 94720-5190.
}

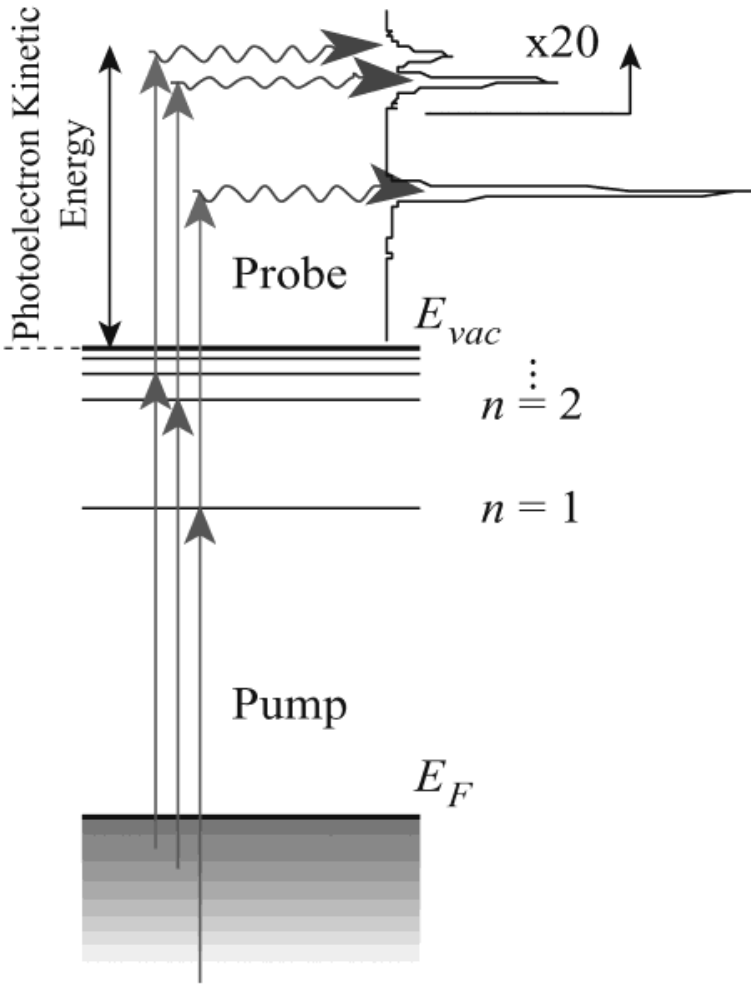

Figure 1. TPPE. The vertical arrows show the excitation of electrons from the occupied states of the substrate, i.e., below the Fermi energy $E_{F}$, to $n=1,2, \ldots$ IPS by the pump pulse $(2 h v)$, and their successive photoemission by the probe pulse $h v$ some time delay $\Delta t$ later. The measured kinetic energy spectrum reflects the energy and population of the IPS.

for two-dimensional solvation, which provides a qualitative description of events that lead to the dynamic change in photoelectron kinetic energy follows the discussion of the experimental findings. 
TPPE was used to populate image potential states (IPS) associated with the interface and to observe the subsequent electron dynamics. The IPS are bound by the attractive interaction between an electron outside of a surface and the surface polarization that it induces. The resultant onedimensional Coulomb potential supports an infinite series of hydrogenic states. For metal surfaces, the energies of IPS in electronvolts are given by

$$
E_{n}=V_{0}-\frac{0.85}{(n+a)^{2}}
$$

where $n$ represents the principal quantum number and $a$ is the quantum defect parameter. ${ }^{27,28}$ The series converges to $V_{0}$, which for a uniform surface is equal to the vacuum energy $E_{\mathrm{vac}}$. Image potential state electrons reside only a few angstroms outside of the interface, making them sensitive to changes in the interfacial electrostatic potential. It has been shown that the surface electronic structure modified by the overlayer also changes the energy and lifetime of the IPS. ${ }^{20,29}$ The static change of the work function upon adsorption of a uniform layer can be taken into account by varying $V_{0} \cdot{ }^{28}$ It will be demonstrated that the time-dependent energies of the IPS reflect adsorbate molecular dipole reorientation that occurs in the presence of the electron. Inside a bulk solvent, molecular reorientation in response to an excess electron is termed electron solvation. Analogously, the collective molecular motion of the adsorbate layer in response to the image potential electron will be termed two-dimensional electron solvation. This solvation is markedly different in the alcohol's first monolayer and in further amorphous multilayers, which reflects the complex interplay of adsorbate-adsorbate and adsorbate-substrate interactions.

\section{Experimental Section}

A detailed description of the experimental apparatus can be found elsewhere. ${ }^{21}$ An initial pump pulse of 344-368 nm was obtained by frequency doubling of the $687-736 \mathrm{~nm}$ probe pulse, which was generated by an optical parametric amplifier (OPA) that was pumped by a Ti:sapphire regenerative amplifier seeded by a Ti:sapphire oscillator. Experiments with $401 \mathrm{~nm}$ pump and $802 \mathrm{~nm}$ probe wavelengths were also performed by utilizing the output of the regenerative amplifier and its second harmonic without the use of the OPA. The typical instrument function exhibits a Gaussian profile with a full width at half-maximum of $\sim 100$ fs.

Aldrich spectra grade anhydrous methanol, 1-propanol, and 1-butanol as well as $99+\%$ reagent grade 1-pentanol were used for the experiment after repeated freeze-pump-thaw purification. Deuterated species $99.8 \%$ methanol- $d\left(\mathrm{CD}_{3} \mathrm{OD}\right)$ and $98 \%$ 1-butanol- $d\left(\mathrm{C}_{4} \mathrm{H}_{9} \mathrm{OD}\right)$ were also obtained from Aldrich. Vapors were introduced via a leak valve into the ultrahigh vacuum chamber that contained a $\mathrm{Ag}(111)$ crystal. Methanol overlayers were grown at $120 \mathrm{~K}, 1$-propanol at $150 \mathrm{~K}, 1$-butanol at 170$180 \mathrm{~K}$, and 1-pentanol at 165-170 K. Exposure times and pressures were converted to langmuir exposures $(1$ langmuir $=$ $10^{-6}$ Torr $\cdot$ s uncorrected for the gauge factors), and the resulting systems were characterized by the TPPE kinetic energy spectra taken with a zero time delay between the laser pulses while the detector was positioned at the surface normal. In coveragedependent studies, a constant bias was applied between the sample and the detector to compensate for the contact potential. The change in the work function was determined by fitting the energy spacings observed at zero time delay for the image potential state series to eq 1 , and adjusting the bias until the

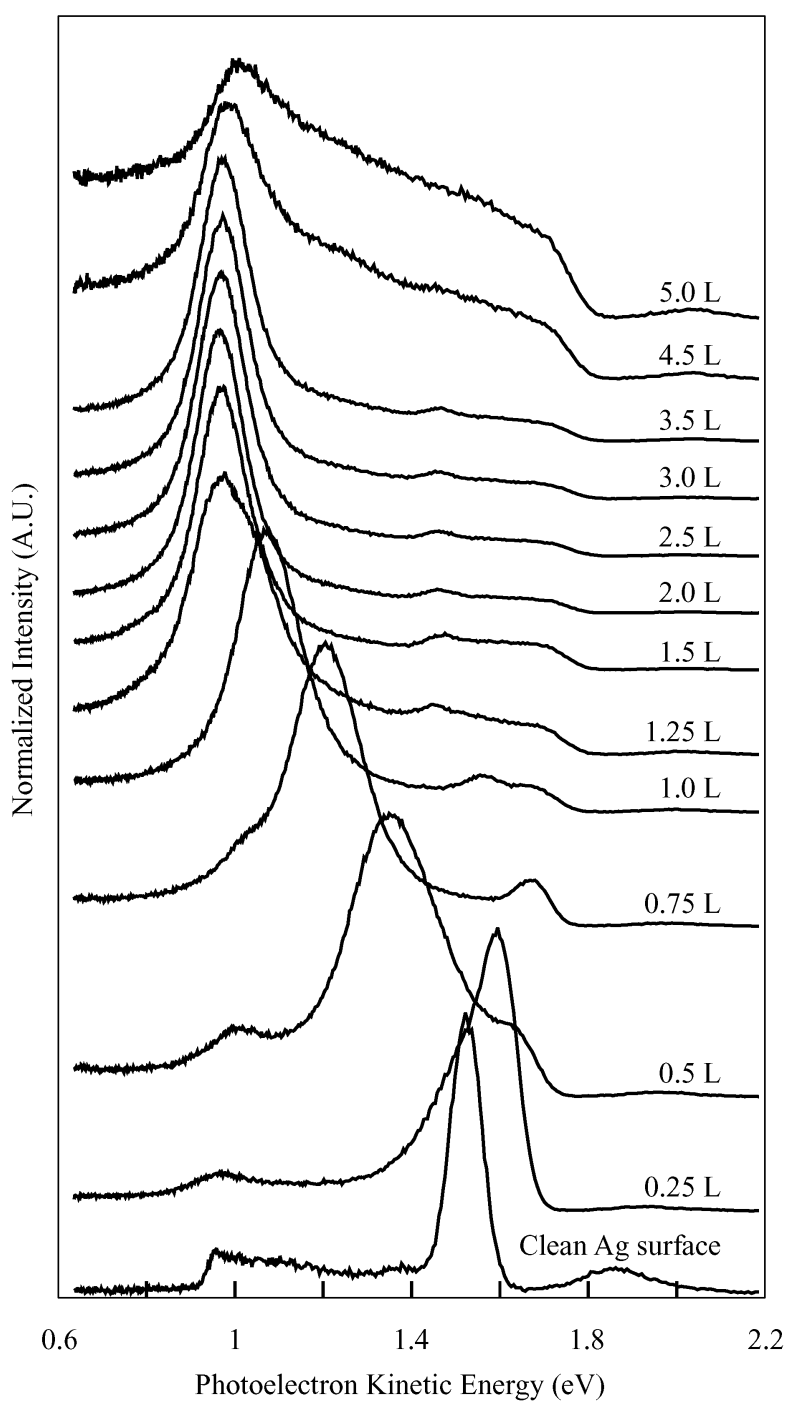

Figure 2. Normalized spectra as a function of exposure of the Ag(111) crystal to methanol vapor, in langmuirs (L) uncorrected for iongauge sensitivity, at $120 \mathrm{~K}$ probed at $725 \mathrm{~nm}$. Exposures of 0.5 langmuirs of methanol quench the clean surface features. At $\sim 1.5$ langmuirs, the methanol has formed a monolayer. From 1.5 to 5 langmuirs, the IPS broaden and decrease in amplitude, showing that the growth of methanol/Ag(111) is not layer-by-layer.

measuring binding energies matched the eq 1 prediction. This produced work function changes consistent with those measured for alcohol adsorption on $\operatorname{Ag}(110) .{ }^{30}$ In time-resolved dynamics scans, when the energies of the IPS were changing, the bias corresponded to field-free propagation as determined from the IPS progressions at zero delay time. Low energy electron diffraction (LEED) determined the amount of long-range periodicity in the layer. The LEED electron beam severely perturbed the adsorbate layers in seconds (methanol) to minutes (1-pentanol), disrupting the diffraction pattern. For this reason, all TPPE dynamics were acquired prior to LEED experiments.

Layer Characterization. Figure 2 presents TPPE spectra as a function of methanol exposure. The clean $\operatorname{Ag}(111)$ surface state and $n=1$ image potential state are quenched by exposures $<0.5$ langmuirs. The new series of peaks increases in intensity, shifts to lower kinetic energy, and sharpens as more methanol is deposited on the surface up to total exposures $\sim 2$ langmuirs. These peaks are consistent with a new series of IPS, with $n=$ 1 and $n=2$ clearly visible. The coverage-dependence of the IPS suggests that the methanol layer initially covers the surface diffusely because of low adsorbate mobility, becoming a 
TABLE 1: Population and Solvation Dynamics of Alcohols/Ag(111)

\begin{tabular}{|c|c|c|c|c|c|c|c|}
\hline & \multirow{2}{*}{$\begin{array}{l}\text { exposure range } \\
\text { (langmuir) }\end{array}$} & \multirow[b]{2}{*}{ peak } & \multicolumn{2}{|c|}{ solvation dynamics ${ }^{a}$} & \multirow{2}{*}{$\begin{array}{c}\text { population dynamics } \\
\text { lifetime ( } \mathrm{fs})\end{array}$} & \multicolumn{2}{|c|}{ static work function } \\
\hline & & & solvation energy $(\mathrm{eV})$ & solvation time $(\mathrm{fs})$ & & measured $(\mathrm{eV})$ & literature $^{27}(\mathrm{eV})$ \\
\hline methanol & $1-3$ & 1 & $0.05(0.04)$ & & $80(90)$ & -1.24 & -1.15 \\
\hline$>\mathrm{ML}$ & & 2 & 0.1 & 280 & 250 & & \\
\hline methanol & $3-5$ & 1 & 0.11 & 780 & 1500 & & \\
\hline$\gg \mathrm{ML}$ & & 2 & 0.25 & 900 & 1500 & & \\
\hline propanol & & 1 & $0.02(0.01)$ & 190 & $100(440)$ & -0.98 & -0.98 \\
\hline$>\mathrm{ML}$ & & 2 & $0.19(0.12)$ & $510(630)$ & $250(240)$ & & \\
\hline butanol ML & $0.8-1.5$ & 1 & $0.01(0)$ & 180 & $160(115)$ & -0.86 & -0.88 \\
\hline butanol & $1-1.4$ & 1 & $0.03(0.01)$ & $260(130)$ & $140(110)$ & & \\
\hline$>\mathrm{ML}$ & & 2 & $0.22(0.15)$ & $530(440)$ & $>500(660)$ & & \\
\hline & & 3 & $0.01(0.01)$ & & $>600(>600)$ & & \\
\hline butanol & $1.2-2.4$ & 1 & 0.027 & 190 & $>1500^{c}$ & & \\
\hline$\gg \mathrm{ML}$ & & 2 & 0.06 & 230 & $>1000^{c}$ & & \\
\hline & & 3 & 0.17 & 400 & $>1000^{c}$ & & \\
\hline pentanol ML & & 1 & $0.1(0.07)$ & $260(200)$ & $140(>200)$ & -0.83 & -0.81 \\
\hline
\end{tabular}

${ }^{a}$ The solvation dynamics were fit to the function $S(t)=A \exp (-t / \tau)+E$. The factor $A$ represents the solvation energy. $\tau$ represents the exponential solvation time constant, and $E$ is the final state energy. Values for corresponding $n=2$ states are included in parentheses. ${ }^{b}$ The popluation dynamics can be fit to an exponential decay time except where noted. ${ }^{c}$ The population dynamics of these states are nonexponential. These estimates provide a lower bound of the lifetime.

completed layer at exposures $\sim 1.5$ langmuirs. ${ }^{31-36}$ The $\sim 1.5$ langmuir exposure also leads to a sharp LEED pattern. Together, the LEED and TPPE indicate that this coverage corresponds to an ordered monolayer.

Further exposure to methanol vapor (3 to 5 langmuirs) does not lead to the appearance of additional peaks indicative of a well-defined second layer. ${ }^{20}$ Instead, it causes gradual broadening and degrading of the monolayer TPPE peaks and also diminishes the LEED pattern. No layer-by-layer growth is observed for methanol/Ag(111), unlike other molecules previously studied with TPPE. ${ }^{20}$ This is consistent with the conclusion, drawn from temperature programmed desorption (TPD) experiments, that the first monolayer of alcohols is ordered and further layers form an amorphous multilayer regime. ${ }^{30,37}$

Although the $\Delta t=0 \mathrm{fs}$ spectra for methanol/Ag(111) seem quite insensitive to the coverage over a broad range of exposures (1.5-4 langmuir), its effect is pronounced in the dynamics. The layers have been assigned to three general coverage regimes based on the characteristics of the dynamics (discussed below). These are, namely, a complete monolayer (ML), a monolayer plus some patches of multilayer ( $>$ ML), and a multilayer coverage $(\gg \mathrm{ML})$ which has quenched all monolayer IPS features. 1-Pentanol, 1-butanol, and 1-propanol data have been similarly grouped. Table 1 contains a summary of the range of exposures that lead to each coverage regime. At the lowest exposures, predominantly ML structures are grown, and at the highest exposures only the $\gg$ ML coverage is obtained. At intermediate exposures, the $>$ ML coverage appears as a superposition of the monolayer and mulitlayer features.

In contrast with the peak desorption temperatures obtained from TPD, ${ }^{30,37}$ the difference between the maximum adsorption temperatures for the monolayer and multilayer regimes of each alcohol appears to be vanishingly small. Within the experimental control over temperature and pressure available, a complete monolayer free of multilayer patches could not be obtained for methanol and 1-propanol. These difficulties controlling the layer growth are somewhat ameliorated with longer alkyl chain lengths, as with the ML coverage of 1-butanol and 1-pentanol. For alcohols, the strong interaction between adsorbates is, in part, the consequence of intermolecular hydrogen bond formation. From this point of view, hydrogen bonding should be less important in longer-chain alcohols and the growth of clean monolayer structures might be expected.

Two-Dimensional Electron Solvation Dynamics. For the range of coverages and systems examined and characterized

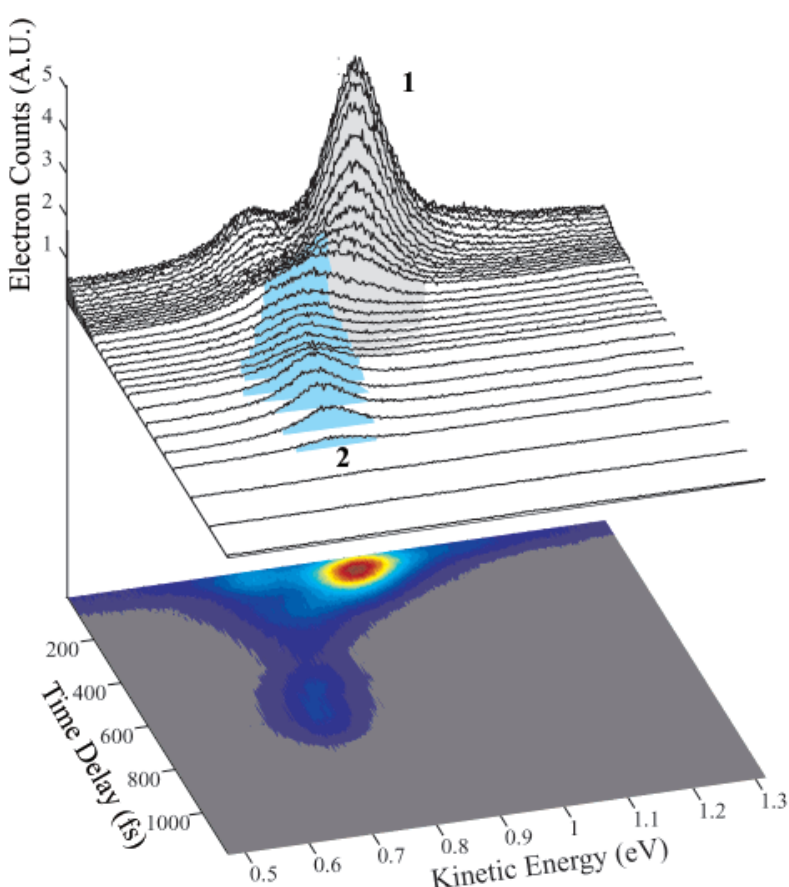

Figure 3. Methanol $>$ ML $n=1$ dynamics at $120 \mathrm{~K}$ and $725 \mathrm{~nm}$. The initial IPS has a short lifetime and shifts very little as a function of time delay. A feature at early times and on the low kinetic energy side of the monolayer IPS has a lifetime limited to the pump-probe correlation and is attributed to the substrate. At long delay times, $600 \mathrm{fs}$, a new signal appears at $0.7 \mathrm{eV}$. This is assigned to multilayer coverages on top of the monolayer.

above, the IPS photoelectrons exhibit dynamically decreasing kinetic energies as a function of pump-probe time delay. This energy relaxation is the result of solvation of the IPS electrons by the polar adsorbate. In general, the solvation energy is greater for the multilayer regime than for the monolayer, although the multiplicity of IPS series for $>$ ML and $\gg$ ML, with each series possessing different population and energy dynamics, complicates the trend. For certain coverages one can observe that $n=$ 1 and $n=2$ states have nearly identical rates of energy relaxation, which indicates that the mechanism of the solvation is a decrease in the local work function. A detail description of the local work function effect will be presented in the discussion section.

The dynamics of the IPS at $>$ ML methanol/Ag(111) are depicted in Figure 3. The most intense IPS (colored gray) shows 


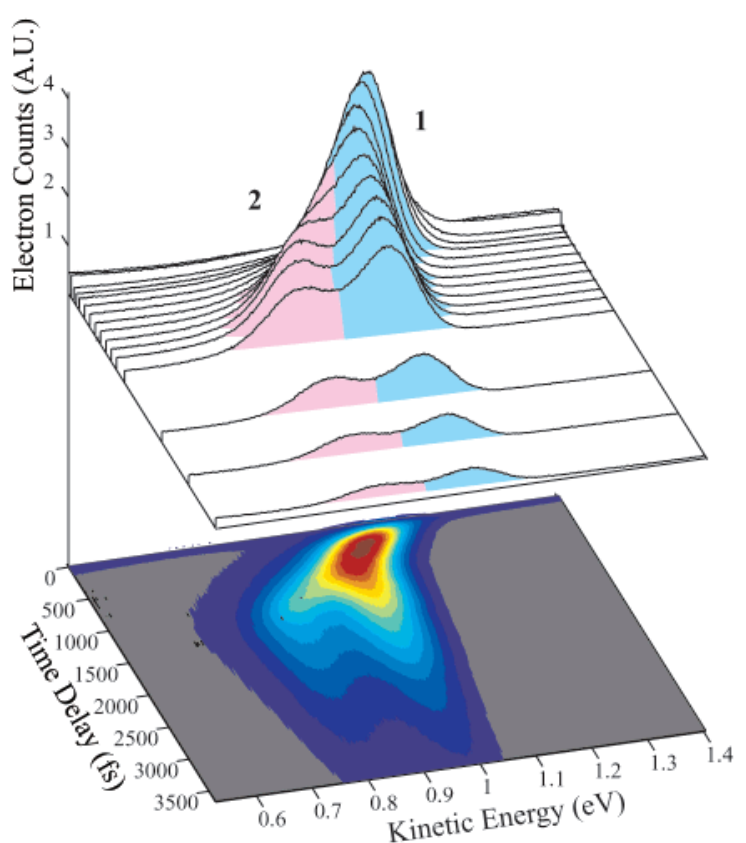

Figure 4. Methanol $\gg$ ML $n=1$ dynamics probed at $710 \mathrm{~nm}$. The ML IPS features have been quenched, as shown by the paucity of signal at $\Delta t=0 \mathrm{fs}$. The multilayer IPS have long rise times (signal maximum appears at $500 \mathrm{fs}$ ) and shift to lower kinetic energies by 0.1 and 0.25 $\mathrm{eV}$, respectively. The two features and their distinct dynamics are caused by different local environments the IPS may sample.

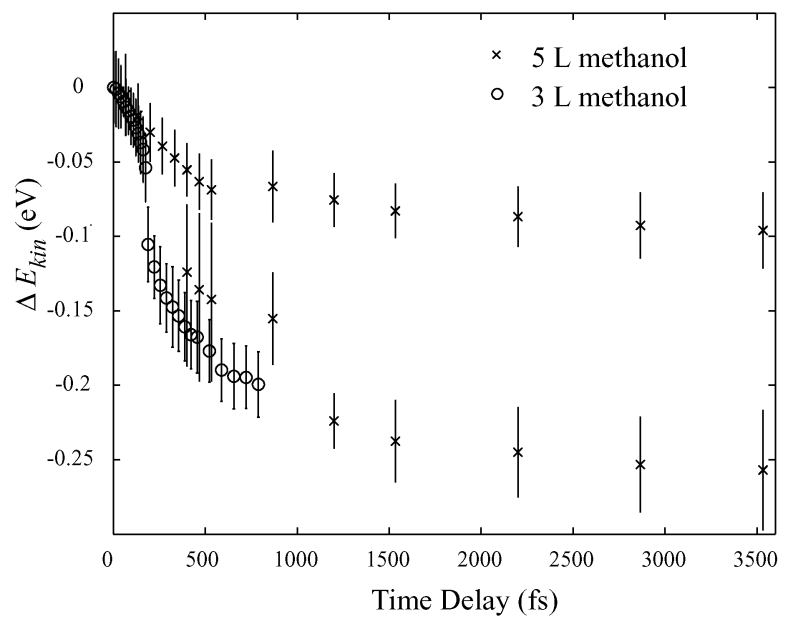

Figure 5. Change in $n=1$ kinetic energy vs $\Delta t$ comparing $>\mathrm{ML}$ (3 langmuir) and $\gg$ ML (5 langmuir) coverages of methanol. The probe wavelengths used for the $>$ ML and $\gg$ ML coverages are 725 and 710 $\mathrm{nm}$, respectively. Multiple $n=1$ IPS are observed for each system. The dynamic shift in kinetic energies represents the two-dimensional solvation of the electron at a methanol/Ag(111) interface.

a short rise time ( $\sim 50 \mathrm{fs})$ and a short lifetime ( $80 \mathrm{fs})$. These attributes are characteristic of ordered monolayers. ${ }^{20}$ In its limited lifetime at the surface, the monolayer IPS shifts to lower kinetic energy by $\sim 0.05 \mathrm{eV}$.

For layers $\gg$ ML, (Figure 4) all peaks in the spectra assigned to $n=1$ states possess very long rise times and lifetimes of several picoseconds. Lifetimes of this magnitude indicate that the alcohol layers effectively decouple the IPS from the metal substrate, shifting the IPS electron density outside of the adsorbate layer into the vacuum. ${ }^{29}$ Most notably, these electronic states have a delay dependent kinetic energy, which decreases by $0.1 \mathrm{eV}$ with an $\sim 800 \mathrm{fs}$ exponential time constant (colored blue) and $0.25 \mathrm{eV}$ with a $900 \mathrm{fs}$ time constant (colored pink).

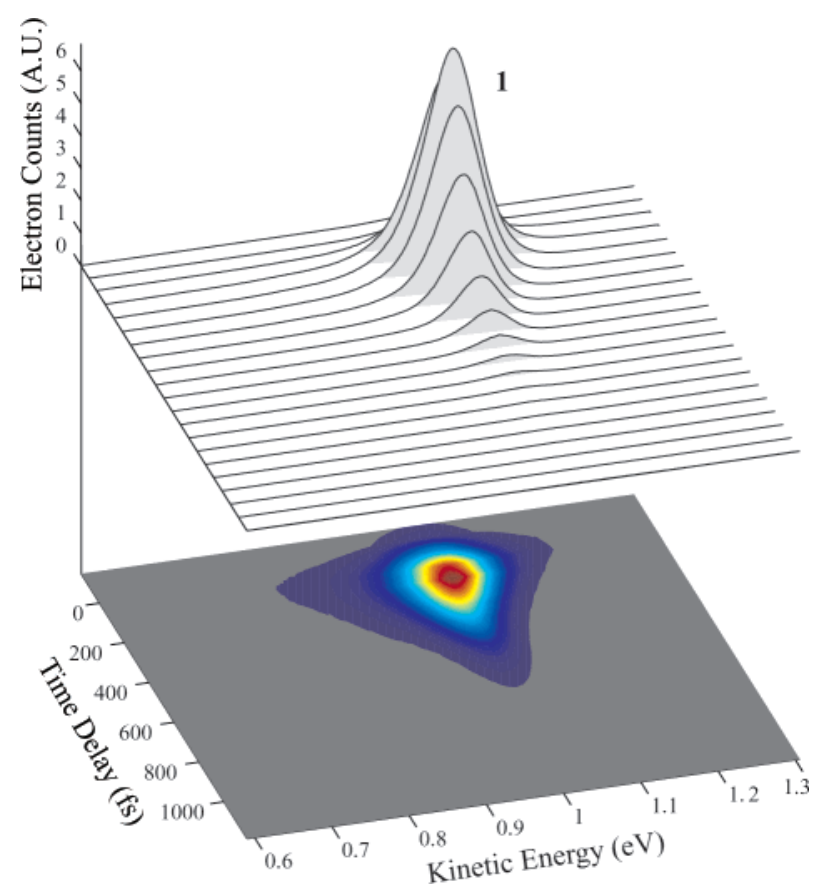

Figure 6. Butanol ML $n=1$ solvation dynamics probed at $687 \mathrm{~nm}$. The energies of the ML IPS shift very little over their limited lifetime at the surface.

The time-dependent peak positions of the $>$ ML and $\gg$ ML states are given in Figure 5.

In Figure 3, one may observe at longer delay times, $600 \mathrm{fs}$, and low kinetic energy, $0.7 \mathrm{eV}$, a small feature that also has a very long rise and decay time and shifts considerably over the course of the dynamics. This feature seems quite similar to the multilayer IPS and is thus assigned to patches of multilayer over the monolayer. No dosage procedure successfully grew a pristine monolayer of methanol/Ag(111) or 1-propanol/Ag(111), though this was achieved with 1-butanol/Ag(111) and 1-pentanol/ $\operatorname{Ag}(111)$ (see below).

The $\Delta t=0$ fs spectra of 1-butanol/Ag(111) show behavior very similar to those of the methanol interface as a function of exposure. The dynamics of three coverages of 1-butanol appear in Figures 6-9. The IPS series characteristic of the monolayer (Figure 6) has relatively short rise and decay times, while the $\gg$ ML IPS have much longer ones (Figure 7). The values of these time constants are summarized in Table 1. The intermediate coverage $(>$ ML) shows a superposition of the high and low coverage regimes in the TPPE spectra (Figure 8). Present are monolayer electronic states (colored gray), which are intense, though short-lived, as well as states that increase in amplitude for hundreds of femtoseconds and do not decay for several picoseconds. The former shift by only $0.01 \mathrm{eV}$ to lower kinetic energy, while the latter states (colored blue) shift as much as $\sim 0.2 \mathrm{eV}$ with the pump-probe delay. At this intermediate coverage, the sharpness of the lines and the good signal-tonoise ratio allow one to observe that the nascent $n=1$ has a companion $n=2$ IPS, and these two features move synchronously to lower kinetic energies (Figure 9). This coverage also evidences a third set of IPS peaks at low kinetic energy that have low initial intensity but grow with time. Neither $n=1$ nor $n=2$ of this last series (colored pink) shifts substantially before (or after) the more intense multilayer peaks overlap them. Both are ascribed to a heterogeneous multilayer where different local environments cause different IPS energies and dynamics.

An intriguing feature of the dynamics of the intermediate coverage of 1-butanol is the time-dependent shift of both $n=$ 


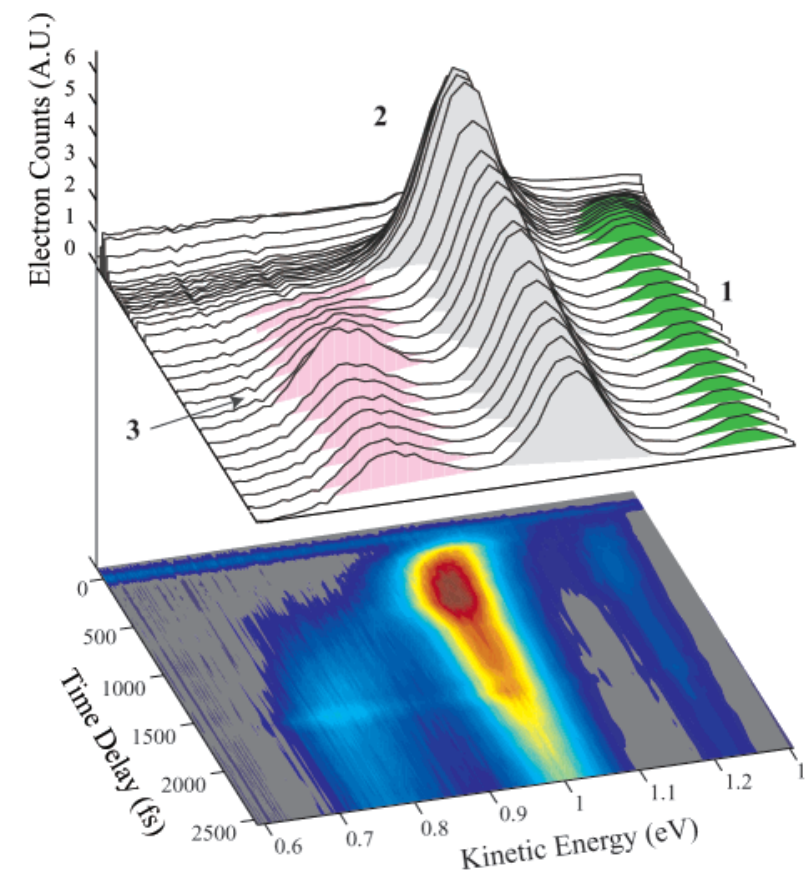

Figure 7. Butanol $\gg$ ML $n=1$ solvation dynamics probed at 688 $\mathrm{nm}$. All features observed exhibit slower population dynamics than those from the ML coverage. State 3 exhibits the largest solvation response: $0.17 \mathrm{eV}$ total relaxation.

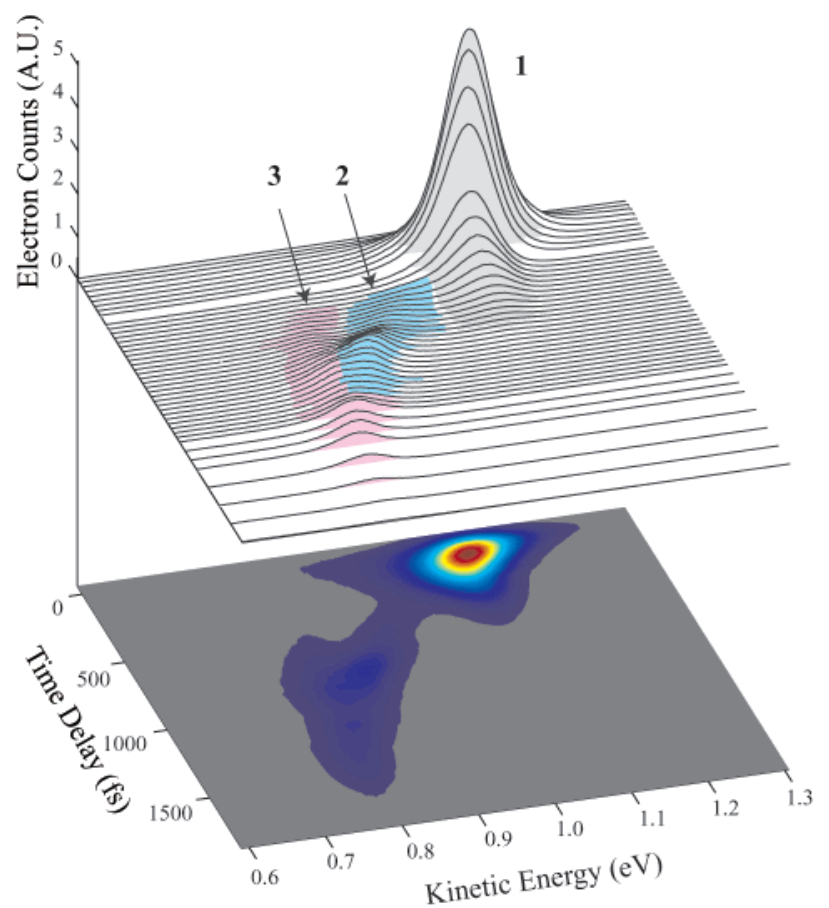

Figure 8. Butanol solvation dynamics of $>$ ML $n=1$ probed at 687 $\mathrm{nm}$. The initial signal (gray) is the ML IPS. The two features that increase in intensity (blue and pink) are characteristic of multilayer growth. The peak colored blue shifts to lower kinetic energy by $\sim 0.2 \mathrm{eV}$.

1 and $n=2$ IPS. In particular, despite the complex range of dynamics appearing in Figure 7, one observable does not change for all IPS series, namely the energy separation between the peaks in each progression (Figure 10b). For each series, the $n$ $=1$ and $n=2$ energies can be fit to eq 1 with the same quantum defect parameters throughout the entire dynamics.

Experiments with the isotope-substituted 1-butanol- $d\left(\mathrm{C}_{4} \mathrm{H}_{9}\right.$ $\mathrm{OD})$ and methanol- $d\left(\mathrm{CD}_{3} \mathrm{OD}\right)$. show dynamics indistinguish-

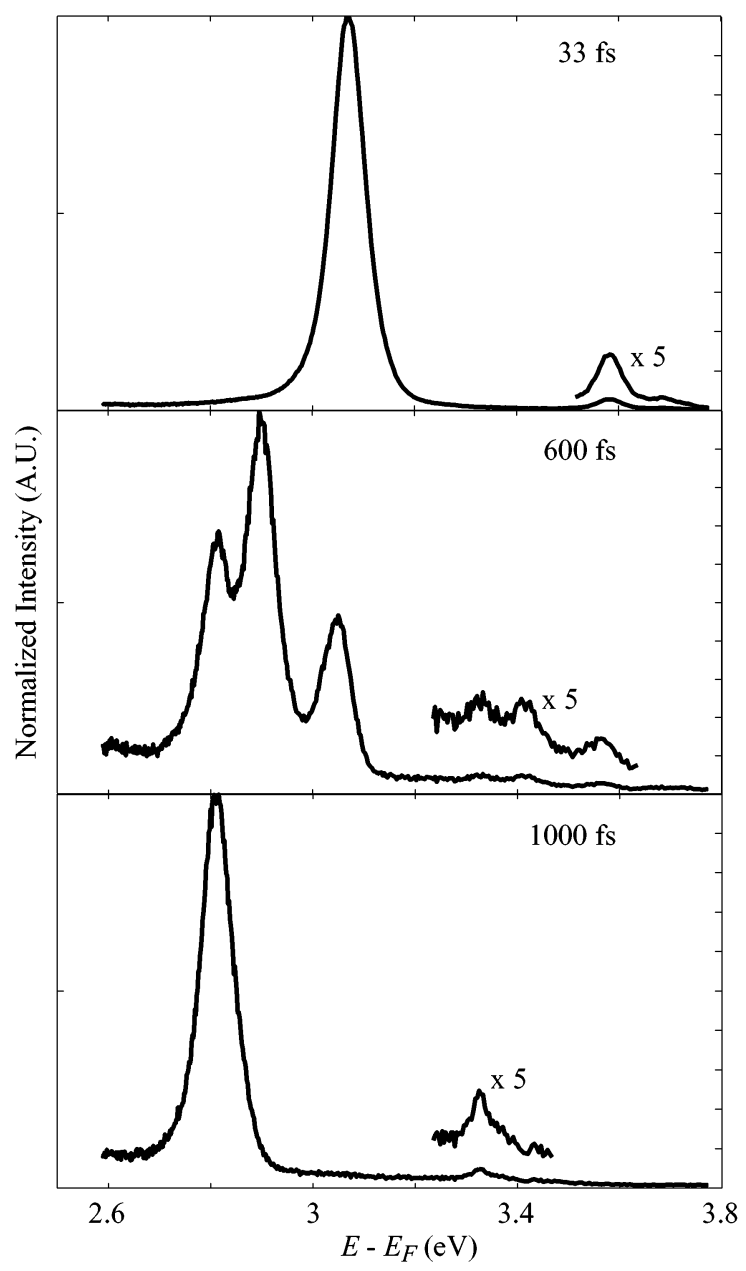

Figure 9. Butanol $>$ ML TPPE spectra at three delay times with all $n$ $=1$ and $n=2$ IPS shown. The probe wavelength is $687 \mathrm{~nm}$.

able from the protonated analogues, within the experimental error. In addition, 1-propanol/Ag(111) behaves very similarly to methanol and 1-butanol (Table 1). The low coverage ML of 1-pentanol was also isolated and examined with TPPE (Table 1).

\section{Discussion}

The TPPE dynamics of IPS at alcohol/Ag(111) interfaces show a great deal of complexity - typical dynamics involve several spectral features with different dynamic energies and different population dynamics. This may be due in part to different coverages appearing on the surface simultaneously. Common features possessed by the higher coverages of adsorbates studied are (i) a single IPS progression is observed immediately after the states are populated by the pump pulse; (ii) at least one additional IPS series appearing in the spectra at later times; (iii) the energies of the IPS shift to lower kinetic energy as a function of pump-probe time delay. As will be shown, the simultaneous appearance of multiple series of IPS as well as the dynamical change in kinetic energies results from different local work functions ${ }^{38}$ whose time dependence is caused by dynamical reorientation of the adsorbate molecules induced by the IPS electron.

The amplitudes of the peaks reflect the population dynamics of the system and their analysis is beyond the scope of the present paper. Briefly, the excitation process leads to the population of a broad band for each IPS, each band corresponding to the distribution of different momenta parallel to the 

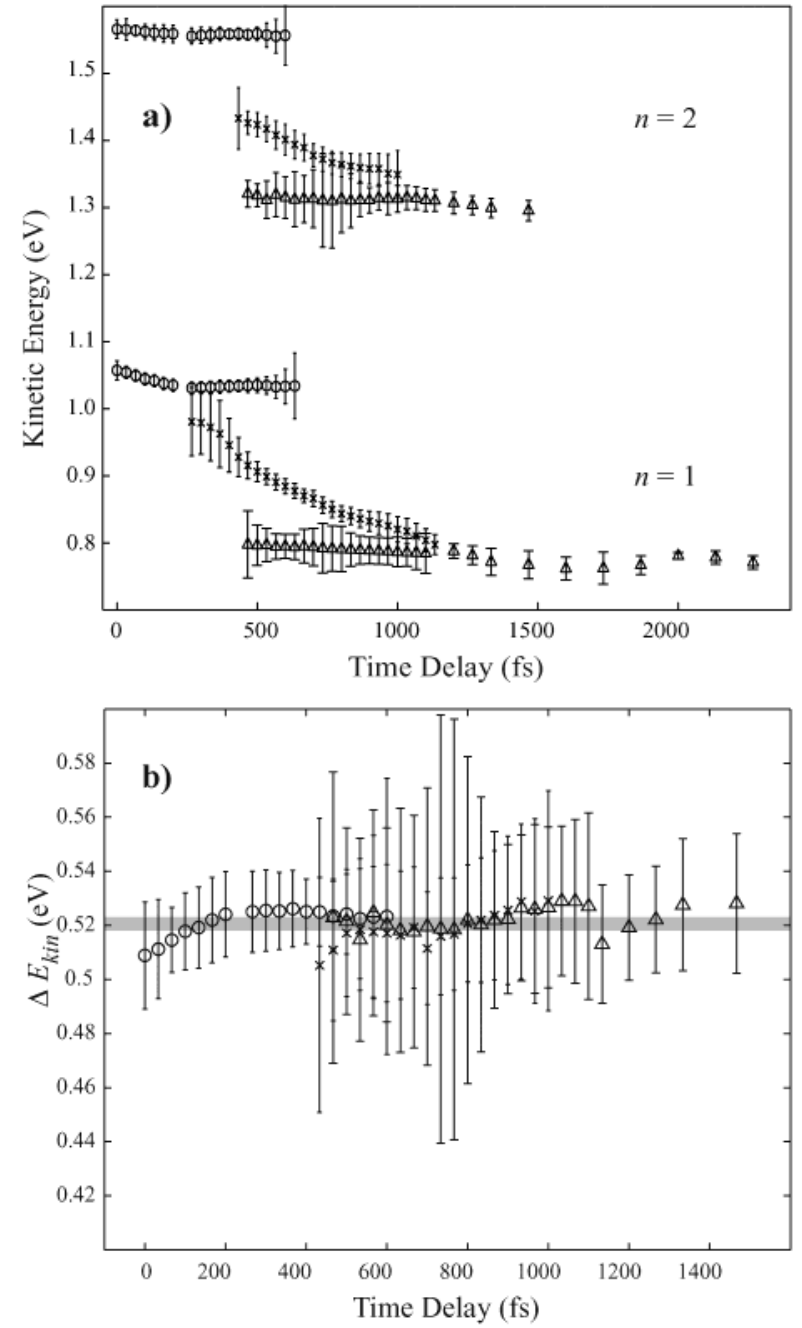

Figure 10. (a) Peak positions vs $\Delta t$ for $>$ ML coverage butanol/Ag(111) probed at $687 \mathrm{~nm}$. The $n=1$ states are accompanied by $n=2$ states which shift to lower kinetic energies synchronously. (b) Kinetic energy difference between $n=2$ and $n=1$ photoelectrons. Because the spacing between the states of each IPS series does not change over the course of the dynamics, the solvation is assigned to a shift in the local work function due to the rotation of the butanol dipole moments.

surface. Only the lowest parallel momentum states can be seen when detecting electrons photoemitted along the surface normal (the main features in the photoelectron kinetic energy spectra in Figures 3 and 4 and 6-8). Population relaxation mechanisms include (i) electron-hole recombination by tunneling back to the metal, ${ }^{20}$ (ii) inelastic scattering from higher parallel momentum states into ones of lower momentum, ${ }^{22,39}$ and (iii) relaxation between bands of different electronic states. ${ }^{2,40}$ Some combination of these processes leads to the population of the additional electronic states that can be seen in Figures 3, 7, and 8 at later delay times. Although a careful analysis can separate all population relaxation processes for relatively simple systems, ${ }^{22}$ the complexity of the alcohol/Ag(111) spectra make this analysis difficult. The present article, instead, focuses on characterization of the time-dependent peak energies, rather than the peak amplitudes. The intermediate coverage $(>\mathrm{ML})$ butanol results presented here give an unambiguous assignment of an energy relaxation mechanism which applies to all of the higher coverage alcohol/Ag(111) interfaces.

Two attributes determine the work function of a metal: the bulk chemical potential and the electrostatic surface potential which arises from the surface dipole layer. ${ }^{38}$ Unlike the surface dipole, the bulk chemical potential does not change measurably with the adsorption of a thin adlayer on the surface of the metal. Photoemission of an electron from a metal requires the electron to cross the metal surface with a characteristic surface dipole that modifies the energy of the electron as it leaves the metal. The electrostatic surface potential experienced by an electron a distance $z$ above the surface can be separated into two contributions:

$$
U(z)=V(z)+V_{\mathrm{IP}}(z)
$$

where $V_{\mathrm{IP}}(z)$ is the potential due to the electronic polarization induced in the substrate and adsorbate by the electron, i.e., the image potential, and $V(z)$ is the electrostatic potential at the interface without the presence of an electron. The $V(z)$ term includes the inherent surface dipole modified by any adsorbate, and the structure of the adsorbate itself. In the case of a polar adsorbate, $V(z)$ accounts for the projection of the molecular dipole onto the surface normal and is thus strongly influenced by the adsorption geometry of the adsorbate. For an ideal homogeneous surface, $V(z)$ is a constant, $V_{0}$, and is the vacuum energy to which the IPS progression converges. The work function can now be defined as the difference between the Fermi energy and $V_{0}$.

Adsorption of an adlayer of alcohol molecules on the Ag(111) surface changes the work function by altering both the electron density distribution of the metal surface and the projection of the molecular dipole onto the surface normal. The static, i.e., $\Delta t=0 \mathrm{fs}$, work function change for each alcohol (Table 1) represents the work function of the layer in its initial configuration before the adsorbate has time to reorganize in response to the excess electron. The constant value of the quantum defect parameters throughout the dynamics (Figure 9) indicates that each set of peaks always represents an IPS series with the same binding energies but which converges to a different $V_{0}$. Previous studies have shown that surface inhomogeneities cause variations in $V(z)$, which, in turn, result in areas with different work functions. ${ }^{31,38}$ These are termed local work functions to emphasize that they correspond to finite areas of the surface. For small patches of surface different from the surrounding area, the work function above the patch is defined as the energy difference between the Fermi level and $V(z)$ close to the surface. For a given patch, "close" means at a distance not greater than twice the radius of the patch parallel to the surface. ${ }^{32}$ The expectation values of the IPS electron in the perpendicular direction from the surface are approximately 3 and $12 \AA$ for $n=1$ and $n=2$, respectively. If the spatial extent of the patch parallel to the surface significantly exceeds the length scale of the first two IPS, then $V(z)$ can be approximated by a constant $V_{0}$ in this region near the surface. The first few IPS in the progression given by eq 1 will then converge not to the vacuum energy but to the local surface potential, $V_{0}$. Multiple local work functions were observed in previous TPPE studies where individual sets of IPS reflected different local static conditions. $^{41}$

An IPS electron only a few angstroms outside the surface significantly perturbs the equilibrium structure of the butanol layer. The molecules respond to the perturbation by rotating the positive end of their molecular dipoles toward the electron, i.e., by solvating it. This reorientation results in an increased projection of the molecular dipole along the surface normal and in a reduction of the work function. The interaction strength between the electron and butanol molecules depends on the degree of electronic spatial localization parallel to the surface. Similar to other systems, ${ }^{1,20,22}$ the initially excited electron is 

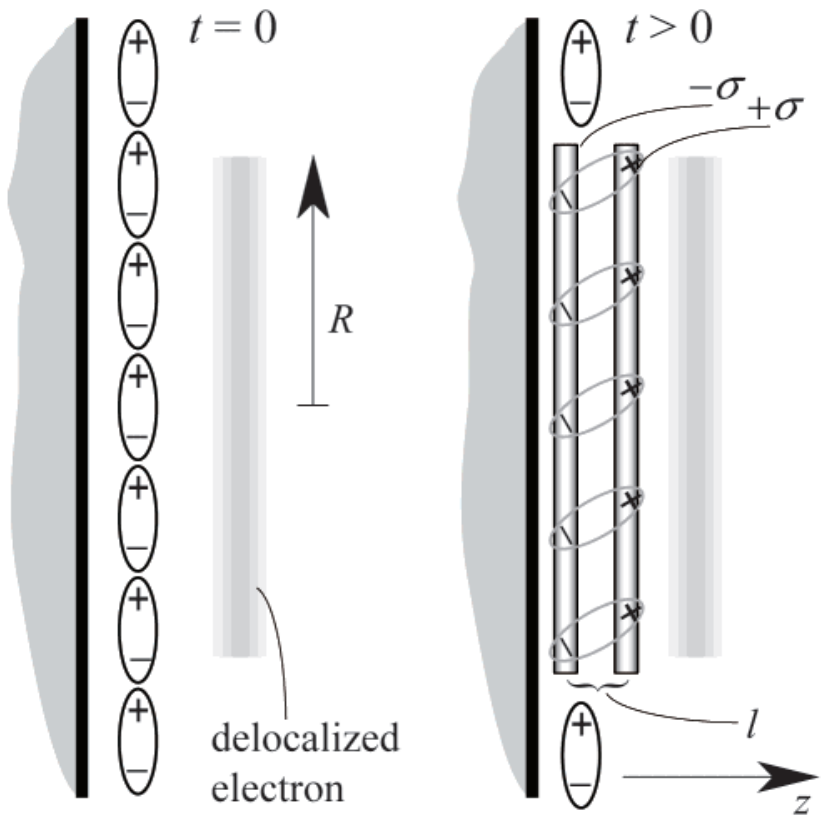

Figure 11. Model of the alcohol molecular dipoles on a surface. Orientation of the molecular dipoles with respect to the surface normal modifies the surface dipole. In the proposed model, two parallel charged disks represent the charge distribution of a patch of molecular dipoles, and collective rotation of the dipoles is equivalent to a change in the disk separation $l$.

delocalized. Were the electron delocalized over the entire substrate, however, the electron density above the adsorbate layer would be negligible, and the force, which the electron produces on each molecule, would be too small to cause reorientation. A more realistic estimate limits the electron delocalization length to the coherence length of the electron. This length is estimated to be $\sim 100 \AA$ (see discussion below). Thus the dynamic change in work function occurs locally with a size comparable to the $100 \AA$ diameter of the delocalized electrons parallel to the surface.

The coherence length of the electron can be estimated from the momentum dependence of the Lorentzian line width in angle-resolved $\mathrm{TPPE}^{2}{ }^{2}$ in which photoemitted electrons are detected along directions not parallel to the surface normal and therefore possess nonzero parallel momentum, $p_{\|}$:

$$
p_{\|}=\left(2 E_{\mathrm{kin}} m_{\mathrm{e}}\right)^{1 / 2} \sin \theta
$$

where $E_{\text {kin }}$ is the kinetic energy of the electron, $m_{\mathrm{e}}$ is the mass of the electron, and $\theta$ is the angle between the surface normal and the detector normal. The analysis assumes a Voight profile for the line shape, with the Gaussian width accounting for the inhomogeneity of the system. ${ }^{42}$ In momentum space, the portion of the Lorentzian width of the Voight profile due to processes parallel to the surface may be estimated from

$$
\Gamma_{\|}=\Gamma\left(p_{\|}\right)-\Gamma(0)
$$

where $\Gamma(0)$ is the Lorentzian width measured at $p_{\|}=0 .{ }^{22}$ Making the assumption that only scattering processes contribute to $\Gamma_{\|}$, the coherence length, $\Delta x$, of the electron is

$$
\Delta x=\frac{p_{\|} \hbar}{m^{*} \Gamma_{\|}}
$$

where $m^{*}$ is the effective mass of the electron in the delocalized
IPS band. ${ }^{2}$ At $p_{\|}=0.2 \AA^{-1}$ and $\Gamma_{\|}=0.02 \mathrm{eV}$, determined from angle-resolved TPPE of 1-propanol/Ag(111) at $\Delta t=0$ fs, $\Delta x$ is $\sim 100 \AA$.

On the same basis, the electron energy shift for $n=1$ and $n$ $=2$ is the same if it is caused by a $100 \AA$ polarized patch of the adsorbate. A simple model estimates changes in the electrostatic potential caused by an IPS electron induced molecular dipole reorientation. For a surface patch with radius $R$, all positive partial charges, $\delta^{+}$, of the positive ends of the alcohol molecular dipoles are combined in a positively charged disk of the radius $R$ and charge density $\sigma=\delta^{+} \rho$, where $\rho$ is the two-dimensional concentration of alcohol molecules on the surface. All negative partial charges $\delta^{-}$are included in a negatively charged disk with the charge density $-\sigma$. Rotation of the dipole, which changes the dipole projection on the surface normal, is represented by increasing the separation between the two disks, $l$, as depicted in Figure 10. The model results in the following potential perpendicular to the surface,

$$
\begin{gathered}
V(z)=-V_{0}\left\{\frac{1}{l}\left[\left(R^{2}+z^{2}\right)^{1 / 2}-\left(R^{2}+(z+l)^{2}\right)^{1 / 2}\right]+1\right\} \\
V_{0}=\frac{q l \sigma}{2 \epsilon_{0}}
\end{gathered}
$$

where $V_{0}$ is the magnitude of the change in potential due to dipole rotation, $l$ is the separation between the disks, $z$ is the distance normal to the disks, $q$ is the charge of the electron, and $\epsilon_{0}$ is the vacuum permittivity. To see the short-range behavior of eq $6 \mathrm{a}$, it is useful to expand $V(z)$

$$
V(z)=-V_{0}\left[1-\frac{z+l / 2}{R}+O\left(\frac{(z+l)^{4}}{l R^{3}}\right)\right]
$$

Clearly, if $z \ll R$, the potential can be approximated by a constant, $V_{0}$. As an example, consider 1-butanol. The charge density is obtained using the dipole moment of $6.0 \times 10^{-30}$ $\mathrm{C} \cdot \mathrm{m}$ and an estimated surface area of $50 \AA^{2}$ for each butanol molecule. Given that $l$ cannot exceed the length of the $\mathrm{OH}$ bond $(0 \leq l \leq 1 \AA)$, and $\sigma=1.2 \times 10^{-21} \mathrm{C} / \AA^{2}$, it gives the change in work function ranging from $0 \mathrm{eV}$, when all the dipoles are aligned parallel to the surface, to $0.6 \mathrm{eV}$ when the dipoles are aligned normal to the surface. The largest measured change of $\sim 0.25 \mathrm{eV}$ for electron solvation by butanol lies well within this range. As a photoemitted IPS electron departs from the surface, the influence of the polarized patch on the electron decreases and $U(z)$ converges to the vacuum energy for large values of $z$. At the same time, the polarized patch relaxes back to the unperturbed state. For a photoemitted electron kinetic energy of $1 \mathrm{eV}$, which is typical in a TPPE experiment, the electron will escape from the local surface potential $V(z)$, within the first $100 \mathrm{fs}$ after photoemission. This implies that the photoelectron will be only slightly influenced by the change in surface potential as the butanol molecules relax back to their unperturbed orientation.

Similar to results observed in the current experiment, invariance of solvation dynamics to deuteration has been observed in other solvation experiments. . $^{3,43,44}$ The effects of deuteration depend critically on the solvent modes responsible for solvation and may be influenced by the nature of the probe solute. ${ }^{43,44}$ Isotope effects should be most apparent in the high-frequency librational modes of the solvent, as demonstrated by experiments on excess electrons in liquid water that probed the solvent response at sub-50 fs time scales. ${ }^{43}$ The present TPPE experi- 
ments do not have sufficient time resolution to observe differences in relaxation rates occurring on similar time scales.

The most pronounced solvation measured for alcohols/Ag(111) appears in higher coverage, multilayer structures, and very little energy relaxation occurs in the monolayers. This is in marked contrast to nitriles on $\operatorname{Ag}(111) .^{2}$ The nearly constant energy of the monolayer IPS strongly suggests that the crystalline alcohol monolayers are much less responsive to the electron than the higher layers due to their stronger interaction with the substrate, i.e., $\operatorname{Ag}(111)$. In some configurations of the higher coverages, however, e.g. those that generate IPS series $\mathbf{2}$ in Figure 8, the alcohols are more mobile, perhaps because of an incomplete hydrogen bond network with dangling $-\mathrm{OH}$ bonds. At the same time, a different configuration, represented by IPS series 3 in Figure 8, shows very little dynamic shift but appears at the same final energy as series 2 . This suggests that some percentage of the surface might already be in a nearly optimal orientation to accommodate the excess electron.

\section{Conclusions}

Time-resolved TPPE has been utilized to study solvation dynamics at two-dimensional interfaces. Collective reorientation of a dipolar adsorbate in the presence of an IPS electron results in dynamic reduction of the local work function which is manifest in the observed dynamic changes of the photoelectron kinetic energies. Similar to the reorganization of a bulk liquid in response to a solute that typifies solvation, the observed reorganization of the adsorbate layer represents electron solvation at the interface. Thus, monitoring the time dependence of the photoelectron energy provides direct access to dynamics of two-dimensional solvation. Monolayers of alcohols show very little energy relaxation, corresponding to their rigid crystalline state. Amorphous, multilayer alcohols present several timedependent features that correspond to heterogeneous environments. Although a detailed understanding of the dynamics is incomplete, these findings demonstrate the power of timeresolved TPPE for studying two-dimensional solvation dynamics at interfaces.

Acknowledgment. This work was supported by the Director, Office of Energy Research, Office of Basic Energy Sciences, Chemical Sciences Division of the U.S. Department of Energy, under Contract No. DE-AC03-76SF00098. The authors acknowledge the National Science Foundation support for specialized equipment used in the experiments described herein.

Note Added after ASAP Posting. This article was released ASAP on 11/13/2002 with an error in eq 3 . The correct version was posted on 11/14/2002.

\section{References and Notes}

(1) Ge, N.-H.; Wong, C. M.; Lingle, R. L., Jr.; McNeill, J. D.; Gaffney, K. J.; Harris, C. B. Science 1998, 279, 202.

(2) Miller, A. D.; Bezel, I.; Gaffney, K. J.; Garrett-Roe, S.; Liu, S. H.; Szymanski, P.; Harris, C. B. Science 2002, 297, 1163.
(3) Gahl, C.; Bovensiepen, U.; Frischkorn, C.; Wolf, M.; Phys. Rev. Lett. 2002, 89, 107402.

(4) Silva, C.; Walhout, P. K.; Yokoyama, K.; Barbara, P. F. Phys. Rev. Lett. 1998, 80, 1086. 465.

(5) Turi, L.; Minary, P.; Rossky, P. J. Chem. Phys. Lett. 2000, 316,

(6) Kloepfer, J. A.; Vilchiz, V. H.; Lenchenkov, V. A.; Germaine, A. C.; Bradforth, S. E. J. Chem. Phys. 2000, 113, 6288.

(7) Stratt, R. M.; Maroncelli, M. J. Phys. Chem. 1996, 100, 12981.

(8) Voth, G. A.; Hochstrasser, R. M. J. Phys. Chem. 1996, 100, 13034

(9) Berg, M.; Vanden Bout, D. A. Acc. Chem. Res. 1997, 30, 65.

(10) Bagchi, B.; Biswas, R. Acc. Chem. Res. 1998, 31, 181.

(11) Bagchi, B.; Fleming, G. R. J. Phys. Chem. 1990, 94, 9.

(12) Karplus, M. J. Phys. Chem. B 2000, 104, 11.

(13) Fleming, G. R.; Cho, M. Аnпи. Rev. Phys. Chem. 1996, 47, 109.

(14) Maroncelli, M.; MacInnis, J.; Fleming, G. R. Science 1989, 243, 1674.

(15) Barthel, E. R.; Martini, I. B.; Schwartz, B. J. J. Phys. Chem. B 2001, 105, 12230

(16) Zimdars, D.; Dadap, J. I.; Eisenthal, K. B.; Heinz, T. F. Chem. Phys. Lett. 1999, 301, 112

(17) Willard, D. M.; Riter, R. E.; Levinger, N. E. J. Am. Chem. Soc. 1998, 120,4151 .

(18) Loughnane, B. J.; Farrer, R. A.; Scodinu, A.; Reilly, T.; Fourkas, J. T. J. Phys. Chem. B 2000, 104, 5421.

(19) Benjamin, I. Chem. Rev. 1996, 96, 1449.

(20) Harris, C. B.; Ge, N.-H.; Lingle, R. L.; McNeill, J. D.; Wong, C. M. Annu. Rev. Phys. Chem. 1997, 48, 711.

(21) McNeill, J. D.; Lingle, R. L.; Ge, N.-H.; Wong, C. M.; Jordan, R. E.; Harris, C. B. Phys. Rev. Lett. 1997, 79, 4645.

(22) Wong, C. M.; McNeill, J. D.; Gaffney, K. J.; Ge, N.-H.; Miller, A.

D.; Liu, S. H.; Harris, C. B. J. Phys. Chem. B 1999, 103, 282.

(23) Petek, H.; Ogawa, S. Prog. Surf. Sci. 1997, 56, 239.

(24) Knoesel, E.; Hertel, T.; Wolf, M.; Ertl, G. Chem. Phys. Lett. 1995, 240,409 .

(25) Bonn, M.; Denzler, D. N.; Funk, S.; Wolf, M.; Wellershoff, S.-S.; Hohlfeld, J. Phys. Rev. B 2000, 30, 1101.

(26) Hofer, U.; Shumay, I. L.; Reuss, C.; Thomann, U.; Wallauer, W.; Fauster, T. Science 1997, 277, 1480

(27) Smith, N. V. Phys. Rev. B 1985, 32, 3549.

(28) Fauster, Th.; Steinmann, W. In Electromagnetic Waves: Recent Developments in Research: Photonic Probes of Surfaces; Halevi, P., Ed.; Elsevier: Amsterdam, New York, 1995; Vol. 2, p 349.

(29) Lingle, R. L., Jr.; Ge, N.-H.; Jordan, R. E.; McNeill, J. D.; Harris, C. B. Chem. Phys. 1996, 205, 191.

(30) Zhang, R.; Gellman, A. J. J. Phys. Chem. 1991, 95, 7433.

(31) Fischer, R.; Fauster, Th.; Steinmann, W. Phys. Rev. B 1993, 48, 15496

(32) Stranick, S. J.; Kamna, M. M.; Weiss, P. S. Surf. Sci. 1995, 338, 41.

(33) Velic, D.; Hotzel, A.; Wolf, M.; Ertl, G. J. Chem. Phys. 1998, 109 , 9155.

(34) Gaffney, K. J.; Wong, C. M.; Liu, S. H.; Miller, A. D.; McNeill,

J. D.; Harris, C. B. Chem. Phys. 2000, 251, 99.

(35) Argile, C.; Rhead, G. E. Surf. Sci. Rep. 1989, 10, 277.

(36) Padowitz, D. F.; Merry, W. R.; Jordan, R. E.; Harris, C. B. Phys. Rev. Lett. 1992, 69, 3583.

(37) Jenniskens, H. G.; Dorlandt, P. W. F.; Kadodwala, M. F.; Kleyn, A. W. Surf. Sci. 1996, 357-358, 624.

(38) Wandelt, K. In Thin Metal Films and Gas Chemisorption; Wissmann, P., Ed.; Elsevier: Amsterdam, New York, 1987; p 280.

(39) Hotzel, A.; Wolf, M.; Gauyarq, J. P. J. Phys. Chem. B 2000, 104, 8438.

(40) Roth, M.; Pickel, M.; Jinxiong, W.; Weinelt, M.; Fauster, Th. Phys. Rev. Lett. 2002, 88, 096802.

(41) Fischer, R.; Schuppler, S.; Fischer, N.; Fauster, Th.; Steinmann, W. Phys. Rev. Lett. 1993, 70, 654.

(42) Mukamel, S. Principles of Nonlinear Optical Spectroscopy; Oxford University Press: New York, 1995.

(43) Emde, M. F.; Baltuška, A.; Kummrow, A.; Pshenichnikov, M. S.; Wiersma, D. A. Phys. Rev. Lett. 1998, 80, 4645.

(44) Lee, S.-H.; Lee, J.-H.; Joo, T. J. Chem. Phys. 1999, 110, 10969 